\title{
Defining a stretching and alignment aware quality measure for linear and curved 2D meshes
}

\author{
Guillermo Aparicio-Estrems, Abel Gargallo-Peiró, Xevi Roca*
}

\begin{abstract}
We define a regularized shape distortion (quality) measure for curved high-order $2 \mathrm{D}$ elements on a Riemannian plane. To this end, we measure the deviation of a given $2 \mathrm{D}$ element, straight-sided or curved, from the stretching and alignment determined by a target metric. The defined distortion (quality) is suitable to check the validity and the quality of straight-sided and curved elements on Riemannian planes determined by constant and point-wise varying metrics. The examples illustrate that the distortion can be minimized to curve (deform) the elements of a given high-order (linear) mesh and try to match with curved (linear) elements the point-wise alignment and stretching of an analytic target metric tensor.
\end{abstract}

\section{Introduction}

In the last decades, the utilization of unstructured meshes composed by highly stretched elements and aligned with dominant flow features, such as boundary layers and shock waves, have shown to be very advantageous [1-3]. When compared with uniform refinement or with isotropic meshes with non-uniform sizing, anisotropic meshes lead to a significant reduction on the number of required degrees of freedom to obtain the same approximation accuracy. This allows performing simulations with a significantly reduced, and even unbeatable, computational cost.

The generation of anisotropic meshes requires to determine the location, stretching and alignment of the elements. These features can be prescribed manually with the help of the user interface of a mesh generation environment. They can also be

Guillermo Aparicio-Estrems

Barcelona Supercomputing Center, 08034 Barcelona (Spain),

Abel Gargallo-Peiró

Barcelona Supercomputing Center, 08034 Barcelona (Spain),

*Xevi Roca

Barcelona Supercomputing Center, 08034 Barcelona (Spain), e-mail: xevi.roca@bsc.es 
prescribed imposing point-wise varying metric tensors obtained in an automatic and iterative adaption procedure based on error indicators or estimators [4-6]. Then, an anisotropic mesher [6-9] can be used to match the resolution, stretching and alignment determined by the target metric.

It is standard to use parallelotopes (quadrilaterals and hexahedra) to manually prescribe the alignment and stretching required to capture flow features such as boundary layers. Whereas the flexibility of simplices (triangles and tetrahedra) is the preferred one in automatic adaption iterations. Nevertheless, for both types of elements, the most mature anisotropic mesh generation techniques lead to meshes featuring second order elements such as multi-linear parallelotopes and linear simplices.

The utilization of curved anisotropic meshes composed by third order elements, such as multi-quadratic parallelotopes and quadratic simplices, or piece-wise polynomial elements of higher order has been mainly centered to curve, manually prescribed, straight-sided boundary layer meshes [10-18]. It has not been until recently that the first metric based approaches have been explored to generate anisotropic meshes featuring straight-sided very high-order three dimensional approximations [19], curved quadratic triangles [20], and r-adapted curved high-order $2 \mathrm{D}$ elements [21]. However, no specific efforts have been conducted to check the validity and measure the quality of curved high-order anisotropic meshes considering a prescribed metric tensor.

Our main contribution is to define a regularized shape distortion (quality) to measure the deviation of a given linear or high-order 2D element from the stretching and alignment determined by a target metric. The influence of the target metric on the element quality has only been considered in detail for linear elements $[22,23]$ and not for curved high-order elements [24]. The defined distortion (quality) is suitable to check the validity and the quality of straight-sided and curved elements on Riemannian planes determined by constant and point-wise varying metrics. Furthermore, we illustrate that the distortion can be minimized to curve (deform) the elements of a given high-order (linear) mesh and try to match with curved (linear) elements the point-wise alignment and stretching of an analytic target metric tensor. Specifically, this approach can be used to improve, by curving (deforming) the elements, the alignment and stretching of a mesh obtained with a straight-sided anisotropic mesher.

The rest of the paper is organized as follows. First, in Section 2 we introduce the quality measures for high-order isotropic 2D elements. Next, in Section 3 we present the new quality measure for high-order anisotropic 2D elements. Following, we present several examples to illustrate the capabilities of the proposed measure, Section 4. To finalize, in Section 5 we present the main conclusions and sum up the future work to develop. 


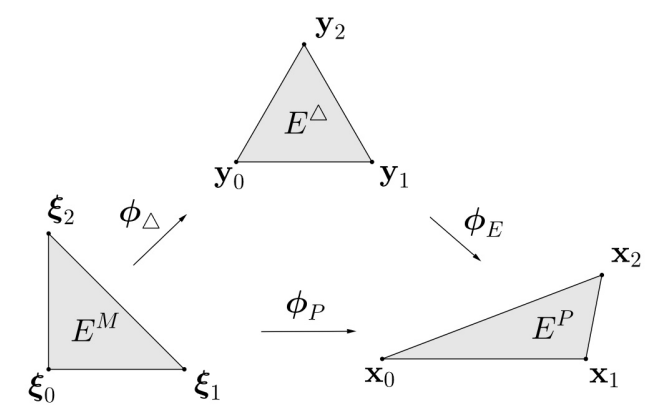

Fig. 1 Mappings between the reference, the ideal and the physical elements in the linear case.

\section{Preliminaries: measures for high-order isotropic 2D elements}

In this section, we review the definition of the Jacobian-based quality measures for linear and high-order isotropic elements. In addition, we introduce the required notation for anisotropic elements.

To define and compute a Jacobian-based measure for linear isotropic triangles [25], three elements are required: the master, the ideal, and the physical, see Figure 1. The master $\left(E^{M}\right)$ is the element from which the iso-parametric mapping is defined. The ideal element $\left(E^{I}\right)$ represents the target configuration which, in the isotropic case, is an equilateral (regular) element $\left(E^{\triangle}\right)$. The physical $\left(E^{P}\right)$ is the element to be measured.

First, the mappings between the ideal and the physical elements through the master element are obtained. By means of these mappings, a mapping between the ideal and physical elements is determined by the composition

$$
\phi_{E}: E^{\triangle} \stackrel{\phi_{\triangle}^{-1}}{\longrightarrow} E^{M} \stackrel{\phi_{P}}{\longrightarrow} E^{P} \text {. }
$$

The Jacobian of this affine mapping (denoted by $\mathbf{D} \phi_{E}$ ) encodes the deviation of the physical element with respect to the equilateral one. Specifically, the distortion measure $\eta$ of the physical element is defined as

$$
\eta\left(\mathbf{D} \phi_{E}\right)=\frac{\operatorname{tr}\left(\left(\mathbf{D} \phi_{E}\right)^{\mathrm{T}} \cdot \mathbf{D} \phi_{E}\right)}{d\left(\operatorname{det}\left(\left(\mathbf{D} \phi_{E}\right)^{\mathrm{T}} \cdot \mathbf{D} \phi_{E}\right)\right)^{1 / d}}=\frac{\operatorname{tr}\left(\left(\mathbf{D} \phi_{E}\right)^{\mathrm{T}} \cdot \mathbf{D} \phi_{E}\right)}{d\left(\operatorname{det}\left(\mathbf{D} \phi_{E}\right)\right)^{2 / d}}
$$

The matrix $\mathbf{D} \phi_{E}$ is computed for linear triangles as

$$
\mathbf{D} \phi_{E}=\mathbf{D} \phi_{P} \cdot \mathbf{D} \phi_{\triangle}^{-1}=\left(\begin{array}{l}
x_{1}-x_{0} \frac{2 x_{2}-x_{1}-x_{0}}{\sqrt{3}} \\
y_{1}-y_{0} \frac{2 y_{2}-y_{1}-y_{0}}{\sqrt{3}}
\end{array}\right),
$$


where

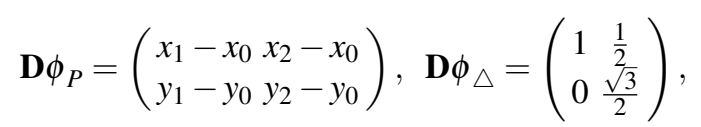

where $\mathbf{x}_{i}=\left(x_{i}, y_{i}\right)$ denotes the coordinates of the physical element $E^{P}$, and where the involved matrices have been written for the master element $E^{M}$ with node coordinates $\left\{\xi_{0}=(-1,-1), \xi_{1}=(1,-1), \xi_{2}=(-1,1)\right\}$, and the ideal element $E^{I}$ determined by the nodes $\left\{\mathbf{u}_{0}=(-1,-1 / \sqrt{3}), \mathbf{u}_{1}=(1,-1 / \sqrt{3}), \mathbf{u}_{2}=(0,2 / \sqrt{3})\right\}$.

The distortion measure in Eq. (1) quantifies the deviation of the shape of the physical element with respect to the regular shape. The measure gets value 1 when the physical element is the equilateral element. It is important to note that it is invariant under translations, rotations, scalings and symmetries. Moreover, it can be regularized to detect inverted elements. From the distortion measure, the quality measure of an element is defined as

$$
q=\frac{1}{\eta}
$$

which takes values in the interval $[0,1]$, being 0 for degenerated elements and 1 for the ideal element.

For high-order [14, 26, 27] and multi-linear [28] elements with non-constant Jacobian, the distortion measure is reinterpreted as a point-wise measure as

$$
\mathscr{N} \phi_{E}(\mathbf{y}):=\eta\left(\mathbf{D} \phi_{E}(\mathbf{y})\right) \forall \mathbf{y} \in E^{\triangle} .
$$

Furthermore, the elemental distortion is defined in $[24,26]$ as

$$
\eta_{E^{P}}:=\frac{\left(\int_{E} \Delta \mathscr{N} \phi_{E}(\mathbf{y})^{2} d \mathbf{y}\right)^{1 / 2}}{\left(\int_{E} \Delta 1 d \mathbf{y}\right)^{1 / 2}}
$$

and its quality $q_{E^{P}}$ follows from Eq. (3).

\section{Measures for curved high-order anisotropic 2D elements}

In this section, we first present a quality measure for linear triangles equipped with a constant metric, see Sec. 3.1. Next, in Sec. 3.2 we analyze the behavior of the defined measure. Finally, in Sec. 3.3 we present the definition of the new quality measure for anisotropic 2D high-order elements. 


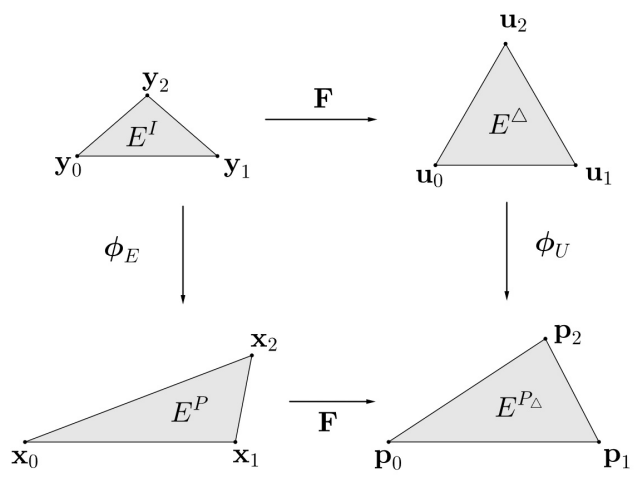

Fig. 2 Mappings between the equilateral, the ideal, the physical and the isotropic physical triangles.

\subsection{Linear elements and constant metric}

To define a measure that quantifies the quality of a given element, it is required to define an ideal element that represents the desired configuration, as detailed in Section 2. In the isotropic case, with metric $\mathbf{M}$ equal to $\lambda \operatorname{Id}$ for $\lambda>0$, the ideal triangle is the equilateral triangle $E^{\triangle}$. For non-isotropic metrics, we describe how to obtain the ideal configuration. Then, we measure the distortion of the physical element comparing it to the ideal element.

We define the ideal element as the element with edges of unit length under the desired metric. To compute this configuration, we first decompose $\mathbf{M}$ as:

$$
\mathbf{M}=\mathbf{F}^{\mathrm{T}} \cdot \mathbf{F} .
$$

Matrix $\mathbf{F}$ can be interpreted as a linear mapping between the space with metric $\mathbf{M}$ and the space with unitary metric. Thus, we define the anisotropic ideal $E^{I}$ as the preimage by $\mathbf{F}$ of the equilateral triangle, see Figure 2. In particular, let $\mathbf{u}_{i}, i=0,1,2$ be the nodes of the equilateral triangle $E^{\triangle}$. Then, we define the nodes of the ideal triangle $E^{I}$ as

$$
\mathbf{y}_{i}=\mathbf{F}^{-1} \cdot \mathbf{u}_{i}, \quad i=0,1,2 .
$$

A direct consequence of the above definition is that the ideal triangle has unit edge lengths in the metric sense.

Once the ideal triangle is defined, we measure the deviation between the ideal and physical elements. Similarly to the approaches for a unitary metric, see Section 2 , in this section we define the distortion between the ideal $E^{I}$ and physical $E^{P}$ elements in terms of the mapping between those elements, $\phi_{E}$.

A priori, we do not know how to compare elements considering the target metric. Nevertheless, we know how to compare elements in the isotropic sense, Sec. 2, and thus we map both elements $E^{I}$ and $E^{P}$ to the same Euclidean space using $\mathbf{F}$, see 


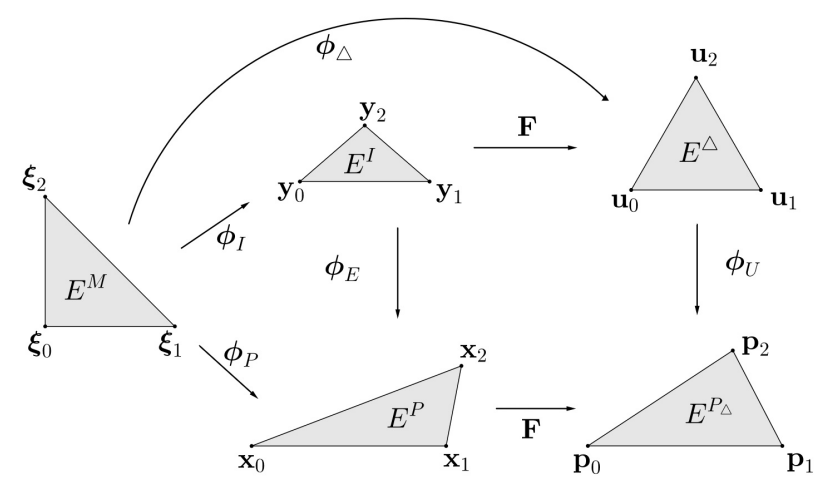

Fig. 3 Mappings between the reference, the equilateral, the ideal, the physical and the isotropic physical triangles.

Figure 2. Then, we compare the image elements $E^{\triangle}$ and $E^{P_{\triangle}}$ using the distortion measure presented in Eq. (1).

Let $E^{P} \triangle$ be the image of the physical triangle $E^{P}$ by $\mathbf{F}$. By construction, the image by $\mathbf{F}$ of the ideal triangle is the equilateral triangle. We measure the distortion between the ideal $E^{I}$ and physical $E^{P}$ elements in terms of the distortion of the mapping between the $E^{\triangle}$ and $E^{P_{\triangle}}$.

Finally, we define the distortion between the physical triangle $E^{P}$ and the ideal triangle $E^{I}$ with respect to the desired metric as the distortion of the matrix $\mathbf{D} \phi_{U}$ :

$$
\eta_{\mathbf{M}}\left(\mathbf{D} \phi_{E}\right)=\eta_{\mathbf{M}}\left(\mathbf{F} \cdot \mathbf{D} \phi_{U} \cdot \mathbf{F}^{-1}\right):=\eta\left(\mathbf{D} \phi_{U}\right)=\frac{\operatorname{tr}\left(\mathbf{D} \phi_{U}^{\mathrm{T}} \cdot \mathbf{D} \phi_{U}\right)}{d \operatorname{det}\left(\mathbf{D} \phi_{U}^{\mathrm{T}} \cdot \mathbf{D} \phi_{U}\right)^{1 / d}}
$$

Remark 1. The distortion presented in Eq. (6) is well defined because it does not depend on the rotations and symmetries of $E^{P_{\Delta}}$. That is, all triangles with the same edge lengths have the same quality. We first show the case for rotations.

The rotation of angle $\theta$ of $E^{P_{\triangle}}$ is the triangle $E^{\tilde{P}_{\triangle}}$ composed by the nodes $\tilde{\mathbf{y}}_{i}=$ $\mathbf{R}(\theta) \cdot \mathbf{y}_{i}, i=0,1,2$. Then

$$
\mathbf{D} \tilde{\phi}_{U}=\mathbf{R}(\theta) \cdot \mathbf{D} \phi_{U}
$$

where $\mathbf{D} \tilde{\phi}_{U}$ is the mapping between the equilateral triangle $E^{\triangle}$ and $E^{\tilde{P}_{\triangle}}$. Next,

$$
\mathbf{D} \tilde{\phi}_{U}{ }^{\mathrm{T}} \cdot \mathbf{D} \tilde{\phi}_{U}=\mathbf{D} \phi_{U}^{\mathrm{T}} \cdot \mathbf{R}(\boldsymbol{\theta})^{\mathrm{T}} \cdot \mathbf{R}(\boldsymbol{\theta}) \cdot \mathbf{D} \phi_{U}=\mathbf{D} \phi_{U}^{\mathrm{T}} \cdot \mathbf{D} \phi_{U} .
$$

By Equations (7), (6) and (3) we conclude that the corresponding qualities are equal. The case for symmetries follows analogously since any symmetry $\mathbf{S}$ satisfies that $\mathbf{S}^{\mathrm{T}} \cdot \mathbf{S}=$ Id. 
Remark 2. To compute the quality measure in Eq. (6) the explicit decomposition of the metric shown in Eq. (5) is not required. Next, we show how to compute the distortion presented in Eq. (6) without decomposing it using matrix $\mathbf{F}$.

First, in Figure 3 we include the master element in the diagram of applications illustrated in Figure 2. Let $\phi_{\triangle}$ be the mapping between the reference and the equilateral triangle. This mapping is equivalent to the composition of the mappings $\phi_{I}$ and $\mathbf{F}$, but it can be directly computed from the coordinates of the master and equilateral triangles, as previously done for the isotropic case in Section 2.

Taking into account the computation of $\mathbf{D} \phi_{\triangle}$ in terms of the node coordinates in Eq. (2), the distortion measure $\eta_{\mathbf{M}}\left(\mathbf{D} \phi_{E}\right)$ can be rewritten without requiring to decompose $\mathbf{M}$. We note that, a priori, right-hand side in Eq. (6) depends on $\mathbf{F}$ since

$$
\mathbf{D} \phi_{U}=\mathbf{D} \phi_{P \triangle} \cdot \mathbf{D} \phi_{\triangle}^{-1}=\mathbf{F} \cdot \mathbf{D} \phi_{P} \cdot \mathbf{D} \phi_{\triangle}^{-1} .
$$

Manipulating Eq. (6) one realizes that there is no explicit dependence on $\mathbf{F}$ :

$$
\begin{aligned}
\mathbf{D} \phi_{U}^{\mathrm{T}} \cdot \mathbf{D} \phi_{U}= & \left(\mathbf{D} \phi_{\triangle}\right)^{-\mathrm{T}} \cdot \mathbf{D} \phi_{P}^{\mathrm{T}} \cdot \mathbf{F}^{\mathrm{T}} \cdot \mathbf{F} \cdot \mathbf{D} \phi_{P} \cdot\left(\mathbf{D} \phi_{\triangle}\right)^{-1} \\
& =\left(\mathbf{D} \phi_{\triangle}\right)^{-\mathrm{T}} \cdot \mathbf{D} \phi_{P}^{\mathrm{T}} \cdot \mathbf{M} \cdot \mathbf{D} \phi_{P} \cdot\left(\mathbf{D} \phi_{\triangle}\right)^{-1} .
\end{aligned}
$$

Thus, we obtain an expression for the distortion that does not require to decompose the metric M:

$$
\eta_{\mathbf{M}}\left(\mathbf{D} \phi_{E}\right)=\eta\left(\mathbf{D} \phi_{U}\right)=\frac{\operatorname{tr}\left(\left(\mathbf{D} \phi_{P} \cdot \mathbf{D} \phi_{\triangle}^{-1}\right)^{\mathrm{T}} \cdot \mathbf{M} \cdot \mathbf{D} \phi_{P} \cdot \mathbf{D} \phi_{\triangle}^{-1}\right)}{d\left(\operatorname{det}\left(\left(\mathbf{D} \phi_{P} \cdot \mathbf{D} \phi_{\triangle}^{-1}\right)^{\mathrm{T}} \cdot \mathbf{M} \cdot \mathbf{D} \phi_{P} \cdot \mathbf{D} \phi_{\triangle}^{-1}\right)\right)^{1 / d}} .
$$

\subsection{Behavior of the metric distortion measure}

In this section, we illustrate the behavior of the shape quality measure corresponding to the distortion measure presented in Eq. (6) for linear anisotropic triangles equipped with a constant metric. We first show the level curves of the quality measure of a triangle when we fix two nodes and we let the third node to move in $\mathbb{R}^{2}$. Second, we analyze the behavior of the measure with respect to the alignment of the element with the metric.

\subsubsection{Level curves of the shape quality measure}

To show the behavior of the level curves of the shape quality measure we consider two cases, the Euclidean or isotropic case when $\mathbf{M}=\mathrm{Id}$ and the anisotropic case when $\mathbf{M}$ has two different eigenvalues. 


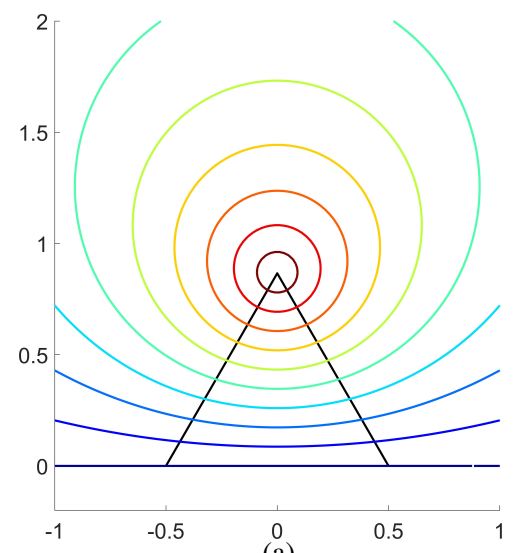

(a)

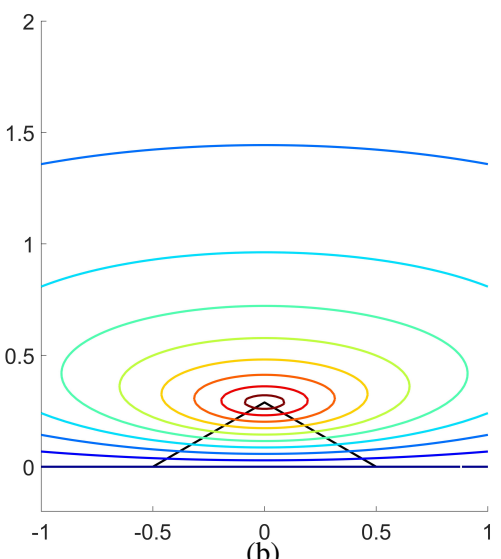

(b)

Fig. 4 Level sets for the shape quality measure: (a) isotropic and (b) anisotropic with metric presented in Eq. (11).

We apply two tests to a triangle, one for each metric. We illustrate the behavior by plotting the level sets in terms of a free node of the triangle. We consider the anisotropic metric given by

$$
\mathbf{M}=\left(\begin{array}{ll}
1 & 0 \\
0 & \frac{1}{h^{2}}
\end{array}\right), h=1 / 3 .
$$

This metric is aligned with the canonical axes and features a stretching ratio of 1 against 3 . Specifically, it is devised to ensure that vectors $(1,0)$ and $(0, h)$ have unit length. The ideal element $E^{I}$ is expected to be an element of height $h$ and base 1. In each test, we consider a free node, keeping the rest of nodes fixed at their original location, and we compute the quality of the element in terms of the location of this node. The free node considered is the vertex node $\mathbf{x}_{2}$.

In Figure 4, we show the contour plots of the quality for each test when the free node is allowed to move in a region of $\mathbb{R}^{2}$. The locus of the points where the element has positive Jacobian, feasible region, is independent of the metric and corresponds to the half-plane $y>0$. As expected, for each metric the optimal node location is different. Furthermore, we can observe that the level sets and the height of the ideal triangle corresponding to the metric of Eq. (11) are more stretched than in the isotropic case. Similarly, the level sets of the quality measure become more stretched as the anisotropy of the metric increases. 


\subsubsection{Alignment dependence of the shape quality measure}

In the second test, we illustrate how the quality measure depends on the alignment between the anisotropy axes and the element. We compute the quality measure of a sequence of physical elements generated rotating the ideal element. We consider the metric presented in Equation (11).

Let $\mathbf{R}(\theta)$ be the rotation at the origin of angle $\theta \in[0,2 \pi)$ which is given by

$$
\mathbf{R}(\theta)=\left(\begin{array}{cc}
\cos \theta & -\sin \theta \\
\sin \theta & \cos \theta
\end{array}\right) .
$$

We define the physical element as the ideal element rotated $\theta$ radians, with nodes $\mathbf{x}_{i}=\mathbf{R}(\theta) \cdot \mathbf{y}_{i}, i=0,1,2$. For each $\theta$ we compute the quality of the corresponding physical element.

In Figure 5, we plot the quality of each physical element with respect to the angle of the rotation applied to the ideal element to generate it. The angle of rotation $\theta$ is represented (in radians) in the $x$-axis and the quality measure is represented in the $y$-axis. The cases $\theta=0, \pi / 2, \pi, 3 \pi / 2$ and $2 \pi$ are marked with a black dot and the corresponding rotations of the ideal element are shown in Figures 5(a), 5(b), 5(c), 5(d) and 5(e), respectively. A rotation of the unit circle in the Euclidean space is mapped as the same ellipse in the metric space, see Figures 5(a)-5(e). We highlight that independently of the applied rotation, the ellipse remains constant. An element with quality one must have the nodes on the ideal ellipse.

In the isotropic case, rotations of the equilateral triangle have quality 1 . In the anisotropic case, when two axes correspond to different eigenvalues of the metric, we observe that the quality oscillates having two maxima and two minima in $[0,2 \pi)$. The maxima are obtained in $\theta=0$ and $\theta=\pi$ and the minima at $\theta=\frac{\pi}{2}$ and $\theta=\frac{3 \pi}{2}$. When $\theta=0$ the rotation $\mathbf{R}(\theta)$ is the identity and $E^{P}=E^{I}$. When $\theta=\frac{\pi}{2}$ then the axes are interchanged (up to sign) and the quality at $\theta=\frac{\pi}{2}$ attains a minimum. The minima are attained when both axes are interchanged (up to sign) and the maxima are attained when the axes coincide with the eigenvectors of the metric (up to sign).

\subsection{Measures for high-order 2D elements on varying metric}

In Section 3.1, we have presented the distortion measure for linear elements equipped with a constant metric. For high-order elements, the Jacobian of the mapping is not constant. In this section, we describe the analogous formulation for high-order elements and for linear elements equipped with a non-constant metric field.

The point-wise distortion measure for an element $E^{P}$ equipped with a metric $\mathbf{M}$, at a point $\mathbf{u} \in E^{\triangle}$ is defined as

$$
\mathscr{N} \phi_{U}(\mathbf{u}):=\eta\left(\mathbf{D} \phi_{U}(\mathbf{u})\right) .
$$




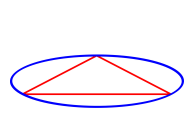

(a)

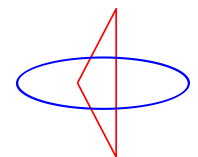

(b)

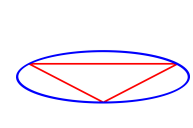

(c)

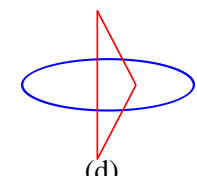

(d)

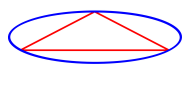

(e)

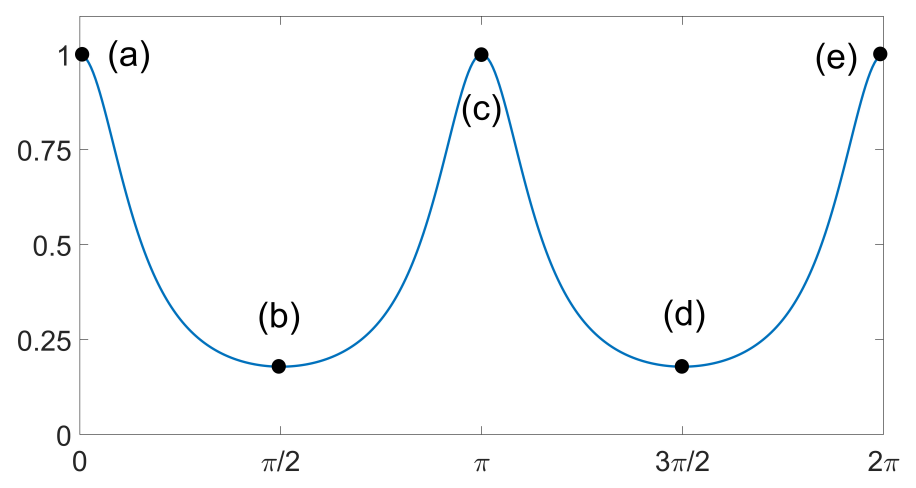

Fig. 5 Shape quality measure of physical elements which are rotations of the ideal element.

Following Eq. (4), the distortion measure for an element $E^{P}$ equipped with a metric $\mathbf{M}$ is defined as

$$
\eta_{\left(E^{P}, \mathbf{M}\right)}=\frac{\left(\int_{E^{\Delta}}\left(\mathscr{N} \phi_{U}(\mathbf{u})\right)^{2} d \mathbf{u}\right)^{1 / 2}}{\left(\int_{E^{\Delta}} 1 d \mathbf{u}\right)^{1 / 2}} .
$$

Eq. (12) can be written in terms of $\xi$ on the master element. That is, the Jacobian of the map $\phi_{U}$ can be written in terms of $\xi$ as:

$$
\mathbf{D} \phi_{U}\left(\phi_{\triangle}(\xi)\right)=\mathbf{F}\left(\phi_{P}(\xi)\right) \cdot \mathbf{D} \phi_{P}(\xi) \cdot\left(\mathbf{D} \phi_{\triangle}(\xi)\right)^{-1}
$$

where

$$
\mathbf{M}\left(\phi_{P}(\xi)\right)=\mathbf{F}\left(\phi_{P}(\xi)\right)^{\mathrm{T}} \cdot \mathbf{F}\left(\phi_{P}(\xi)\right) .
$$

Then, Eq. (12) reads

$$
\eta_{\left(E^{P}, \mathbf{M}\right)}=\frac{\left(\int_{E^{M}}\left(\mathscr{N} \phi_{U}\left(\phi_{\triangle}(\xi)\right)\right)^{2}\left|\operatorname{det} \mathbf{D} \phi_{\triangle}(\xi)\right| d \xi\right)^{1 / 2}}{\left(\int_{E^{M}}\left|\operatorname{det} \mathbf{D} \phi_{\triangle}(\xi)\right| d \xi\right)^{1 / 2}} .
$$

Similarly to Eq. (10), the decomposition of the metric is not required:

$$
\mathbf{D} \phi_{U}\left(\phi_{\triangle}(\xi)\right)^{\mathrm{T}} \cdot \mathbf{D} \phi_{U}\left(\phi_{\triangle}(\xi)\right)=\mathbf{A}(\xi)^{\mathrm{T}} \cdot \mathbf{M}\left(\phi_{P}(\xi)\right) \cdot \mathbf{A}(\xi),
$$

where

$$
\mathbf{A}(\xi):=\mathbf{D} \phi_{P}(\xi) \cdot\left(\mathbf{D} \phi_{\triangle}(\xi)\right)^{-1}
$$


Using the above equation we obtain the final expression on each point $\xi$ of the master element:

$$
\mathscr{N} \phi_{U}\left(\phi_{\triangle}(\xi)\right)=\frac{\operatorname{tr}\left(\mathbf{A}(\xi)^{\mathrm{T}} \cdot \mathbf{M}\left(\phi_{P}(\xi)\right) \cdot \mathbf{A}(\xi)\right)}{d\left(\operatorname{det}\left(\mathbf{A}(\xi)^{\mathrm{T}} \cdot \mathbf{M}\left(\phi_{P}(\xi)\right) \cdot \mathbf{A}(\xi)\right)\right)^{1 / d}} .
$$

In order to detect inverted elements [14,29-31] we regularize the determinant in the denominator of Eq. (14) to

$$
\sigma_{0}=\frac{1}{2}(\sigma+|\sigma|)
$$

where

$$
\sigma=\operatorname{det} \mathbf{D} \phi_{P}(\xi) \operatorname{det} \mathbf{D} \phi_{\triangle}(\xi)^{-1} \sqrt{\operatorname{det} \mathbf{M}\left(\phi_{P}(\xi)\right)} .
$$

Then, the point-wise regularized distortion measure of a physical element $E^{P}$ is defined as

$$
\mathscr{N}_{0} \phi_{U}(\mathbf{u}):=\eta_{0}\left(\mathbf{D} \phi_{U}(\mathbf{u})\right):=\frac{\operatorname{tr}\left(\mathbf{D} \phi_{U}^{\mathrm{T}} \cdot \mathbf{D} \phi_{U}\right)}{d \sigma_{0}^{2 / d}} .
$$

Finally, the regularization of the elemental distortion given in Equation (12) is given by

$$
\eta_{0,\left(E^{P}, \mathbf{M}\right)}:=\frac{\left(\int_{E^{\triangle}}\left(\mathscr{N}_{0} \phi_{U}(\mathbf{u})\right)^{2} d \mathbf{u}\right)^{1 / 2}}{\left(\int_{E^{\Delta}} 1 d \mathbf{u}\right)^{1 / 2}}
$$

and its corresponding quality

$$
q_{0,\left(E^{P}, \mathbf{M}\right)}=\frac{1}{\eta_{0,\left(E^{P}, \mathbf{M}\right)}} .
$$

\section{Results}

In this section, we present several examples to illustrate the main features of the proposed quality measure. Each example features an analytic and point-wise varying metric with continuous first derivatives. For each case, we generate an initial mesh and we measure its quality according to the new measure for anisotropic elements. Next, we optimize the location of the nodes to minimize the element distortion using the framework presented in $[14,32]$.

The formulation to obtain the ideal element described in Section 3.1 can be extended to quadrilaterals in a straight-forward manner by considering the unit square $E^{\square}$ as the ideal element in the Euclidean case [28]. To illustrate the applicability of the presented measures for both triangles and quadrilaterals, each example 
is presented for an initial mesh featuring triangles, and an initial mesh featuring quadrilaterals.

In each case, we present a table summarizing the element quality statistics. Specifically, we show the minimum quality, the maximum quality, the mean quality and the standard deviation of the initial and optimized meshes. We highlight that in all cases, the optimized mesh increases the minimum element quality and it does not include any inverted element. The meshes resulting after the optimization are composed by elements as aligned and stretched as possible to match the target metric tensor. In all figures, the meshes are colored according to the elemental quality, see Eq. (15).

As a proof of concept, a mesh optimizer has been developed in MATLAB using the Optimization Toolbox, the PDE Toolbox and the Symbolic Math Toolbox. The MATLAB prototyping code is sequential (one execution thread), corresponds to the implementation of the method presented in this work. In all the examples, the optimization is reduced to find a minimum of a nonlinear unconstrained multi-variable function, where a trust-region algorithm is used. The stopping condition is set to reach a relative residual smaller than $10^{-8}$.

\subsection{Boundary layer mesh: triangles and quadrilaterals}

In this example, we illustrate how our approach deals with boundary layers. We choose a metric with a geometric grow of the size in the $y$ direction. We consider a rectangular domain $\Omega=[0,1] \times\left[0, y_{\max }\right]$ with the metric

$$
\mathbf{M}(x, y):=\left(\begin{array}{cc}
1 & 0 \\
0 & 1 / h(y)^{2}
\end{array}\right)
$$

where

$$
h(y):=\left\{\begin{array}{cc}
h_{\min }+\gamma y & \text { for } y<y_{0} \\
1-\left(1-h_{0}\right) e^{-r\left(y-y_{0}\right)} & \text { for } y \geq y_{0}
\end{array}, \quad(x, y) \in \Omega,\right.
$$

being $\gamma=1.3$ the growing ratio, $h_{\min }=10^{-3}$ the minimum size in the $y$ direction, and $y_{\max }=5\left(1-h_{\min }\right) / \gamma \approx 3.8423$ the top height of the domain. The parameters $h_{0}=1-10^{-5}$ and $r=\gamma /\left(1-h_{0}\right)=1.3 \cdot 10^{5}$ are chosen to determine a differentiable metric and $y_{0}=\left(h_{0}-h_{\min }\right) / \gamma \approx 0.7685$ is the height of the transition between the anisotropic and the isotropic regions determined by the metric. The metric attains the highest level of anisotropy at $y=0$, with a maximum quantity $1: 1 / h_{\min }$. When $y \geq y_{0}$ the metric is almost isotropic.

We generate two isotropic meshes, one featuring triangles and one featuring quadrilaterals. The unstructured triangular linear mesh is composed by 230 nodes and 394 elements. The structured quadrilateral linear mesh is composed by 208 nodes and 175 elements. The initial isotropic meshes are illustrated in Figures 6(a) and 6(c), coloring the elements according to the quality for anisotropic meshes pre- 


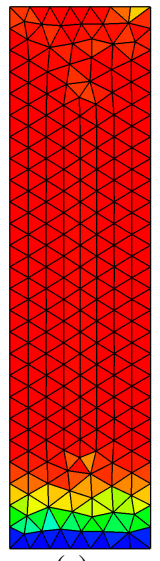

(a)

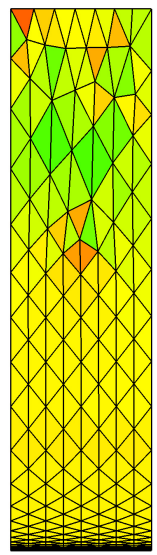

(b)

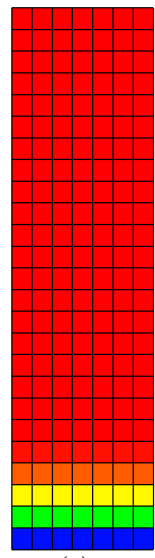

(c)

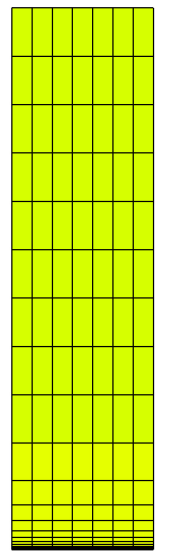

(d)

Fig. 6 Meshes for a rectangle domain featuring a vertical boundary layer metric: (a) initial triangular mesh, (b) optimized triangular mesh, (c) initial quadrilateral mesh, and (d) optimized quadrilateral mesh

Table 1 Quality Statistics for boundary layer case.

\begin{tabular}{lllllll}
\hline Figure & Mesh & Element Type & Min & Max & Mean & Std. Dev. \\
\hline 6(a) & Initial & Triangles & 0.0068 & 1.0000 & 0.9255 & 0.2076 \\
6(b) & Optimized & Triangles & 0.5754 & 0.9046 & 0.7411 & 0.0459 \\
6(c) & Initial & Quadrilaterals & 0.0168 & 0.9733 & 0.9241 & 0.2150 \\
6(d) & Optimized & Quadrilaterals & 0.7097 & 0.7229 & 0.7182 & 0.0063 \\
\hline
\end{tabular}

sented in Eq. (15). We observe that the elements of minimal quality (in blue) lie in the region of higher anisotropy.

For each mesh we optimize the location of the nodes to minimize the distortion, letting the inner nodes move in $\mathbb{R}^{2}$ and the boundary nodes slide on their corresponding boundaries. The optimized meshes are shown in Figures 6(b) and 6(d). The quality statistics of both the initial and optimized meshes are shown in Table 1. The minimum is improved after the optimization process and the standard deviation of the element qualities is reduced. We observe that in the optimized meshes the elements lying in the region $y \geq y_{0}$ have been enlarged vertically to allow the elements located in the region $y<y_{0}$ to be compressed vertically. 


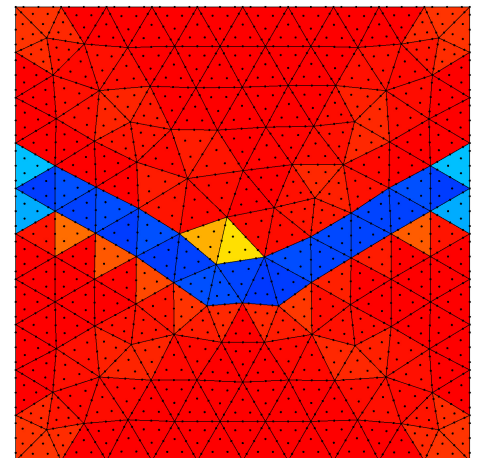

(a)

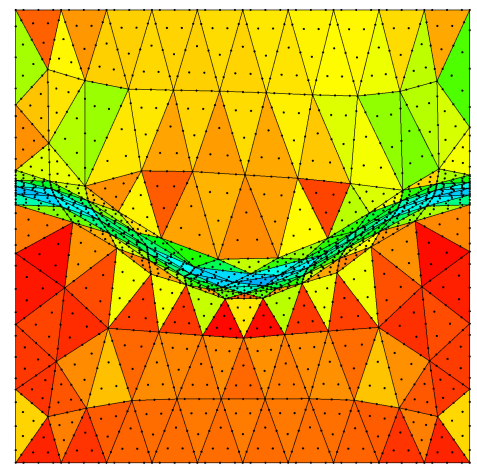

(c)

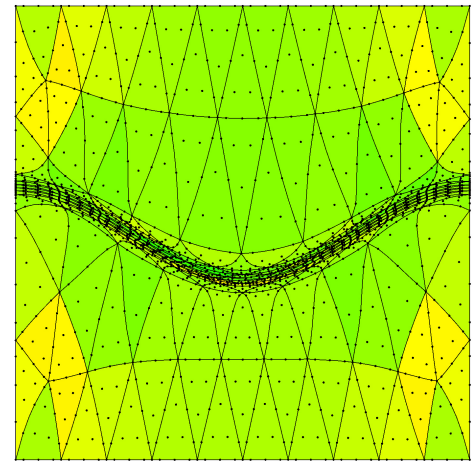

(b)

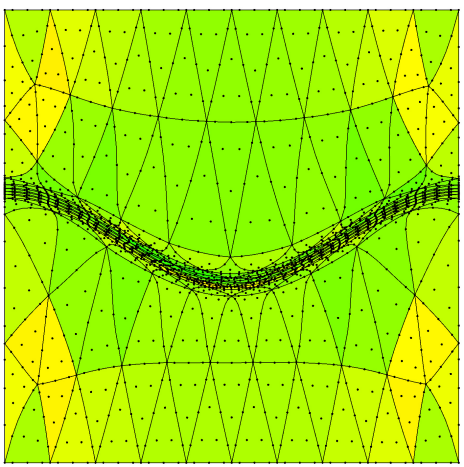

(d)

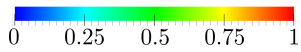

Fig. 7 Triangle meshes of polynomial degree 4: (a) initial straight-sided isotropic mesh, (b) optimized mesh from initial configuration (a), (c) initial straight-sided anisotropic mesh, (d) optimized mesh from initial configuration (c).

\subsection{Curved anisotropy: curved quartic quadrilaterals and triangles}

In this example, we illustrate the behavior for high-order elements of the proposed quality measure and of the optimization framework $[14,32]$ applied to the new developed quality measure. We consider the quadrilateral domain $\Omega:=[0,1]^{2}$ and the metric $\mathbf{M}:=\nabla \varphi^{\mathrm{T}} \cdot \nabla \varphi$ induced by the surface $\varphi$ :

$$
\varphi(\mathbf{x}):=(x, y, z(x, y)), \quad z(x, y):=\tanh (f \cdot h(x, y)),
$$

where

$$
h(x, y)=\frac{1}{\sqrt{4 \pi^{2}+100}} \cdot(10 y-\cos (2 \pi x)-5), \quad(x, y) \in \Omega, f=5 .
$$




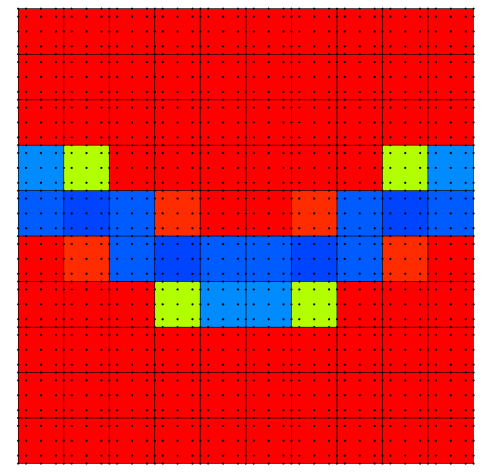

(a)

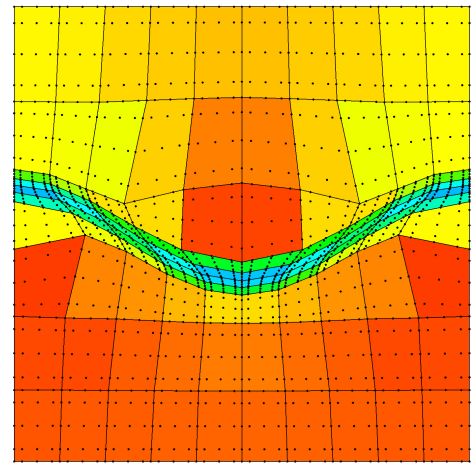

(c)

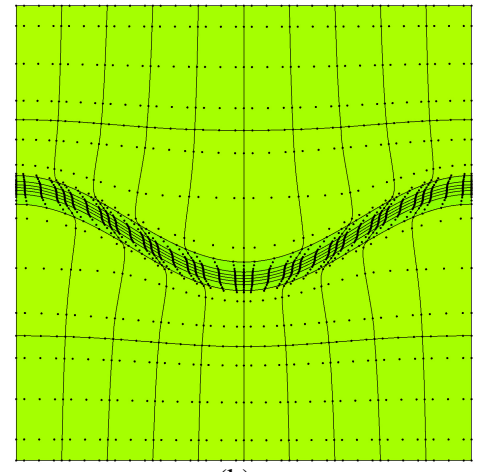

(b)

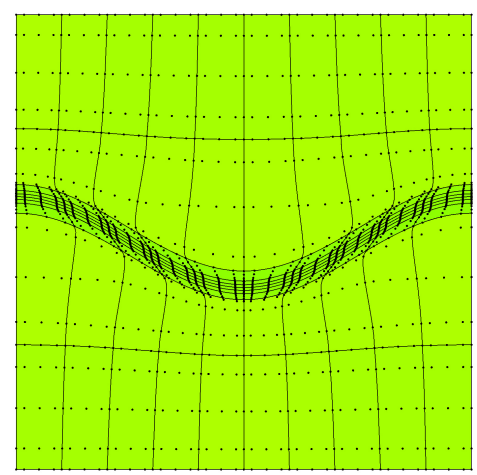

(d)

Fig. 8 Quadrilateral meshes of polynomial degree 4: (a) initial straight-sided isotropic mesh, (b) optimized mesh from initial configuration (a), (c) initial straight-sided anisotropic mesh, (d) optimized mesh from initial configuration (c).

The metric is extracted from [33] and attains the highest level of anisotropy at the curve described by the points $(x, y) \in \Omega$ such that $h(x, y)=0$. The anisotropy ratio of this metric is $1: \sqrt{1+|\nabla z|^{2}}$ and its maximum is given by $1: \sqrt{1+f^{2}}$.

We illustrate this example both for triangles, Figure 7, and quadrilaterals, Figure 8. For each element type, we generate two different initial straight-sided high-order meshes: an isotropic mesh, Figures 7(a) and 8(a), and an anisotropic mesh, Figures $7(\mathrm{c})$ and $8(\mathrm{c})$. The quadrilateral meshes are composed by 1681 nodes and 100 elements and the triangular meshes are composed by 1905 nodes and 228 elements. The anisotropic initial meshes, Figures 7(c) and 8(c), are generated by means of optimizing the linear isotropic mesh and next, increasing the polynomial degree of the linear optimized mesh. We highlight that the two straight-sided anisotropic meshes are better initial configurations to curve the high-order mesh according to the metric. Although they are not optimal meshes of polynomial degree 4 , they have been 
Table 2 Quality Statistics of the meshes of polynomial degree 4 for curved anisotropy.

\begin{tabular}{lcccccc}
\hline Figure & Mesh & Element type & Min & Max & Mean & Std. Dev. \\
\hline $7(a)$ & Initial & Triangles & 0.0569 & 1.0000 & 0.8849 & 0.2824 \\
$7(\mathrm{~b})$ & Optimized & Triangles & 0.5381 & 0.7626 & 0.6570 & 0.0435 \\
$7(\mathrm{c})$ & Initial & Triangles & 0.1726 & 0.9905 & 0.6739 & 0.2308 \\
$7(\mathrm{~d})$ & Optimized & Triangles & 0.5381 & 0.7626 & 0.6570 & 0.0435 \\
$8(\mathrm{a})$ & Initial & Quadrilaterals & 0.0631 & 1.0000 & 0.8408 & 0.3324 \\
$8(\mathrm{~b})$ & Optimized & Quadrilaterals & 0.6384 & 0.6696 & 0.6506 & 0.0130 \\
$8(\mathrm{c})$ & Initial & Quadrilaterals & 0.1852 & 0.9405 & 0.6631 & 0.2417 \\
8(d) & Optimized & Quadrilaterals & 0.6384 & 0.6696 & 0.6506 & 0.0130 \\
\hline
\end{tabular}

obtained from an optimal linear mesh. All the meshes are colored in the figures according to the elemental quality measure presented in Eq. (15).

The optimized meshes for the triangle case are illustrated in Figures 7(b) and 7(d), and for the quadrilateral case are illustrated in Figures 8(b) and 8(d). In the optimized meshes the elements away from the anisotropic region are enlarged vertically whereas the elements lying in the anisotropic region are compressed. For the triangle and quadrilateral cases, the two meshes optimized from the two different initial configurations are equal up to the chosen optimization tolerance. The quality statistics of both the initial and optimized meshes are shown in Table 2. In all the optimized meshes the minimum is improved and the standard deviation of the element qualities is reduced when compared with the initial configuration.

\section{Concluding remarks and future work}

In this work, we have presented a new definition of distortion (quality) measures for linear and high-order planar anisotropic meshes equipped with a point-wise metric. The proposed quality measures the alignment and stretching of the elements according to the given metric. In addition, it is valid for any interpolation degree and allow to detect the validity of a high-order element equipped with a metric. To assess the reliability of the technique, we have first analyzed the behavior of the measure for linear triangles equipped with a constant metric. The tests show that for a given metric the obtained quality measure detects invalid and low-quality configurations and, the alignments and stretching described by the metric.

The defined distortion measure is applied to curve linear meshes to improve the node configuration according to the desired metric. To perform the distortion optimization we use the framework for high-order optimization presented in $[14,32]$. The numerical examples show optimized meshes with an improved alignment and stretching according to the metric. This improvement leads in all the cases to an increase of the minimum elemental mesh quality and a reduction of the standard deviation between the different element qualities. 
Our long term goal is to extend the quality measure for 3D anisotropic meshes. In addition, the quality measure developed in this work is devised to quantify the alignment and the stretching of the mesh according to the target metric. Thus, we would like to extend the proposed measure to also quantify the mesh sizing.

Acknowledgements This project has received funding from the European Research Council (ERC) under the European Union's Horizon 2020 research and innovation programme under grant agreement No 715546. This work has also received funding from the Generalitat de Catalunya under grant number 2017 SGR 1731 . The work of X. Roca has been partially supported by the Spanish Ministerio de Economía y Competitividad under the personal grant agreement RYC-2015-01633.

\section{References}

1. F. Alauzet and A. Loseille. A decade of progress on anisotropic mesh adaptation for computational fluid dynamics. Computer-Aided Design, Elsevier, 72(1):13-39, 2016.

2. C. Gruau and T. Coupez. 3d tetrahedral, unstructured and anisotropic mesh generation with adaptation to natural and multidomain metric. Computer Methods in Applied Mechanics and Engineering, 194(48-49):4951-4976, 2005.

3. P. Frey and F. Alauzet. Anisotropic mesh adaptation for cfd computations. Computer methods in applied mechanics and engineering, 194(48-49):5068-5082, 2005.

4. M. Yano and D. L. Darmofal. An optimization-based framework for anisotropic simplex mesh adaptation. Journal of Computational Physics, 231(22):7626-7649, 2012.

5. A. Loseille and F. Alauzet. Continuous mesh framework part i: well-posed continuous interpolation error. SIAM Journal on Numerical Analysis, 49(1):38-60, 2011.

6. T. Coupez, L. Silva, and E. Hachem. Implicit boundary and adaptive anisotropic meshing. In New Challenges in Grid Generation and Adaptivity for Scientific Computing, pages 1-18. Springer, 2015.

7. F. Hecht. Bamg: bidimensional anisotropic mesh generator. User Guide. INRIA, Rocquencourt, 1998.

8. A. Loseille and R. Lohner. Cavity-based operators for mesh adaptation. In 51st AIAA Aerospace Sciences Meeting including the New Horizons Forum and Aerospace Exposition, page 152, 2013.

9. A. Loseille. Metric-orthogonal anisotropic mesh generation. Procedia Engineering, 82:403415, 2014.

10. O. Sahni, X.J. Luo, K.E. Jansen, and M.S. Shephard. Curved boundary layer meshing for adaptive viscous flow simulations. Finite Elem. Anal. Des., 46(1):132-139, 2010.

11. P.-O. Persson and J. Peraire. Curved mesh generation and mesh refinement using lagrangian solid mechanics. In Proc. 47th AIAA, 2009.

12. T. Toulorge, C. Geuzaine, J.-F. is Remacle, and J. Lambrechts. Robust untangling of curvilinear meshes. Journal of Computational Physics, 254:8-26, 2013.

13. A. Gargallo-Peiró, X. Roca, J. Peraire, and J. Sarrate. Inserting curved boundary layers for viscous flow simulation with high-order tetrahedra. In Research Notes, 22nd Int. Meshing Roundtable. Springer International Publishing, 2013.

14. A. Gargallo-Peiró, X. Roca, J. Peraire, and J. Sarrate. Optimization of a regularized distortion measure to generate curved high-order unstructured tetrahedral meshes. Int. J. Numer. Meth. Eng., 103:342-363, 2015.

15. D. Moxey, M.D. Green, S.J. Sherwin, and J. Peiró. An isoparametric approach to high-order curvilinear boundary-layer meshing. Computer Methods in Applied Mechanics and Engineering, 283:636-650, 2015. 
16. M. Fortunato and P-O. Persson. High-order unstructured curved mesh generation using the winslow equations. Journal of Computational Physics, 307:1-14, 2016.

17. A. Gargallo-Peiró, G. Houzeaux, and X. Roca. Subdividing triangular and quadrilateral meshes in parallel to approximate curved geometries. Procedia Engineering, 203:310-322, 2017.

18. D. Moxey, D. Ekelschot, Ü. Keskin, S. J. Sherwin, and J. Peiró. High-order curvilinear meshing using a thermo-elastic analogy. Computer-Aided Design, 72:130-139, 2016.

19. O. Coulaud and A. Loseille. Very high order anisotropic metric-based mesh adaptation in $3 \mathrm{~d}$. Procedia Engineering, pages 353-365, 2016. Proceedings of the 25th International Meshing Roundtable.

20. T. Coupez. On a basis framework for high order anisotropic mesh adaptation. 203:141-153, 2017. Research Note 26th International Meshing Roundtable.

21. J. Marcon, M. Turner, D. Moxey, S. J. Sherwin, and J. Peiró. A variational approach to highorder r-adaptation. IMR26, 2017.

22. W. Huang and Y. Wang. Anisotropic mesh quality measures and adaptation for polygonal meshes. 2015

23. W. Huang. Measuring mesh qualities and applications to variational mesh adaptation. SIAM J. Sci. Comput., 26(5):1643-1666, 2005.

24. X. Roca, A. Gargallo-Peiró, and J. Sarrate. Defining quality measures for high-order planar triangles and curved mesh generation. In Proc. 20th Int. Meshing Roundtable, pages 365-383. Springer International Publishing, 2012.

25. P. M. Knupp. Algebraic mesh quality metrics. SIAM J. Numer. Anal., 23(1):193-218, 2001.

26. A. Gargallo-Peiró, X. Roca, J. Peraire, and J. Sarrate. Distortion and quality measures for validating and generating high-order tetrahedral meshes. Eng. Comput., 31:423-437, 2015.

27. A. Gargallo-Peiró, X. Roca, J. Peraire, and J. Sarrate. A distortion measure to validate and generate curved high-order meshes on CAD surfaces with independence of parameterization. Int. J. Numer. Meth. Eng., 106(13):1100-1130, 2015.

28. A. Gargallo-Peiró, E. Ruiz-Gironés, X. Roca, and J. Sarrate. On curving high-order hexahedral meshes. In 24th International Meshing Roundtable (IMR24), October 11-14, 2014, Austin, TX, pages 1-5. Elsevier, 2015.

29. L. V. Branets and V. A. Garanzha. Distortion measure of trilinear mapping. application to 3-d grid generation. Numerical linear algebra with applications, 9(6-7):511-526, 2002.

30. E. J. López, N. M. Nigro, and M. A. Storti. Simultaneous untangling and smoothing of moving grids. Int. J. Numer. Meth. Eng., 76(7):994-1019, 2008.

31. J. M. Escobar, E. Rodríguez, R. Montenegro, G. Montero, and J. M. González-Yuste. Simultaneous untangling and smoothing of tetrahedral meshes. Comput. Meth. Appl. Mech. Eng., 192(25):2775-2787, 2003.

32. A. Gargallo-Peiró. Validation and generation of curved meshes for high-order unstructured methods. PhD thesis, Universitat Politècnica de Catalunya, 2014.

33. P. C. Caplan, R. Haimes, D. L. Darmofal, and M. C. Galbraith. Anisotropic geometryconforming d-simplicial meshing via isometric embeddings. Procedia Engineering, 203:141153, 2017. 26th International Meshing Roundtable. 